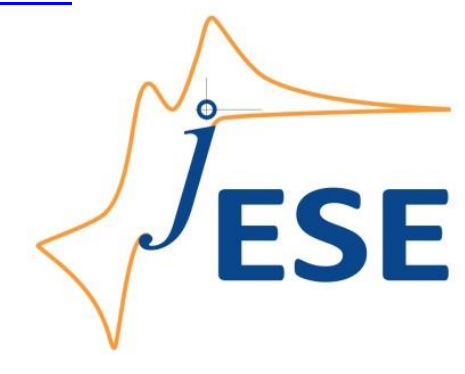

Open Access : : ISSN 1847-9286

www.jESE-online.org

Original scientific paper

\title{
Influence of intensity of ultrasound on morphology and hardness of copper coatings obtained by electrodeposition
}

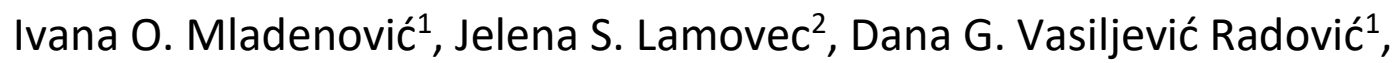 \\ Vesna J. Radojević ${ }^{3}$ and Nebojša D. Nikolić ${ }^{1, \bigotimes}$ \\ ${ }^{1}$ University of Belgrade, Institute of Chemistry, Technology and Metallurgy, Njegoševa 12, 11000 \\ Belgrade,Serbia \\ ${ }^{2}$ University of Criminal Investigation and Police Studies, Cara Dušana 196, Zemun, 11000 Belgrade, \\ Serbia \\ ${ }^{3}$ University of Belgrade, Faculty of Technology and Metallurgy, Karnegijeva 4, 11000 Belgrade, \\ Serbia
}

Corresponding author: ${ }^{\circledR n i k o l i c @ i h t m . b g . a c . r s ; ~ T e l .: ~+381-11-337-03-90 ; ~ F a x: ~+381-11-337-03-89 ~}$

Received: February 8, 2022; Accepted: February 25, 2022; Published: February 27, 2022

\begin{abstract}
The influence of various intensities of ultrasound applied for the electrolyte stirring on morphological and mechanical characteristics of electrolytically produced copper coatings has been investigated. The copper coatings produced by the galvanostatic regime of the electrodeposition from the basic sulphate electrolyte and the electrolyte with added levelling/brightening additives at the low temperature were characterized by scanning electron microscopy (SEM) and atomic force microscopy (AFM) techniques (surface morphology and topography, respectively) and by Vickers microindentation (hardness). The roughness of coatings increased with the increasing intensity of ultrasound, indicating that morphology of the coatings worsened with the enhanced application of ultrasonic waves. This is attributed to the strong effect of ultrasound on hydrodynamic conditions in the near-electrode layer, which is manifested by the increase of share of the activation control in the mixed activation-diffusion control of electrodeposition with increasing the intensity of ultrasound. The concept of "effective overpotential" originally proposed to explain a change of surface morphology in the conditions of vigorous hydrogen evolution is also applicable for a change of morphology of Cu coatings under the imposed effect of ultrasonic waves. Hardness analysis of the coatings showed that an intensity of applied ultrasound did not have any significant effect on the hardness, especially for the Cu coatings produced from the basic sulphate electrolyte.
\end{abstract}

\section{Keywords}

Copper; surface characterization; scanning electron microscopy; atomic force microscopy; topography; composite hardness model 


\section{Introduction}

Copper attracts huge attention from both scientific and technological communities owing to its unique characteristics, such as excellent thermal and electrical conductivities, malleability, catalytic activity, superior mechanical, anticorrosion and antimicrobial features [1-3]. These characteristics of copper enable its wide application in various industries, such as aerospace, automotive, microelectronics, telecommunications, medicine, energy sciences and technologies, etc. For all these purposes, the main methods of $\mathrm{Cu}$ synthesis in different forms are electrodeposition, electroless plating, chemical vapor deposition (CVD), physical vapor deposition (PVD), thermal spray and sputtering techniques [2].

The electrodeposition technique is a very suitable way to obtain copper of desired characteristics suitable for application in above mentioned purposes [4]. Copper is possible to obtain in various morphological forms starting from compact to powdered forms by the easy selection of parameters and regimes of the electrodeposition [4-6]. The technologically important $\mathrm{Cu}$ electrodeposition processes include electroplating with a high aspect ratio $[7,8]$, formation of functional thin films and coatings, and creation of nanoparticles and powders [4,9-15]. Although acid sulphate electrolytes based on copper(II) sulphate and sulphuric acid are the most often used electrolytes for $\mathrm{Cu}$ electrodeposition, some other types, such as cyanide and fluoborate, also found application in the electrodeposition processes [16]. The concentrations of main components of electrolyte but also the addition of specific substances in electrolytes like additives for levelling and brightness, also play an important role in the final quality of electrolytically produced coatings. The other electrodeposition parameters determining the quality of deposits are the temperature of the electrolyte, electrodeposition time (or thickness of deposit), type of used cathode, mixing of electrolyte during electrodeposition process, rate of circulation of electrolyte, etc. [4,10,17-20]. All these parameters affect the quality of coatings produced by both constant (potentiostatic and galvanostatic (DC)) and periodically changing regimes (pulsating overpotential (PO), pulsating current (PC) and reversing current (RC)) of the electrodeposition [4].

Although electrodeposition processes are often performed in silent electrolytes, stirring of electrolyte is desirable to obtain smooth and uniform coatings, especially when electrodeposition process performs by the constant regimes. The most often used ways for electrolyte stirring are magnetic stirring [10] and application of ultrasound [21-23]. It was shown that the application of various ways of electrolyte stirring affects mass transfer, nucleation, and growth, and finally, morphology and structure of electrolytically synthesized coatings.

In this study, we examine the effect of various intensities of applied ultrasound (or power of ultrasound) on the morphology of $\mathrm{Cu}$ coatings produced in the galvanostatic (DC) regime. The two types of electrolytes were analysed: the basic sulphate electrolyte and an electrolyte with the addition of levelling and brightening additives. Mechanical characteristics of the produced $\mathrm{Cu}$ coatings are also considered. Characterization of the $\mathrm{Cu}$ coatings was performed by SEM and AFM (surface characterization) techniques and by Vickers microindentation (mechanical characterization).

\section{Experimental}

Copper was electrodeposited galvanostatically at a current density of $50 \mathrm{~mA} \mathrm{~cm}^{-2}$ in an open cell of cylindrical shape. The $\mathrm{Cu}$ coatings thicknesses from $16.6 \mu \mathrm{m}$ were electrodeposited at the low temperature $\left(t=7.0 \pm 1.0^{\circ} \mathrm{C}\right)$ from the following electrolytes:

a. $240 \mathrm{~g} / \mathrm{I} \mathrm{CuSO}_{4} \cdot 5 \mathrm{H}_{2} \mathrm{O}+60 \mathrm{~g} / \mathrm{I} \mathrm{H}_{2} \mathrm{SO}_{4}$; (electrolyte I) and

b. $240 \mathrm{~g} / \mathrm{I} \mathrm{CuSO}_{4} \cdot 5 \mathrm{H}_{2} \mathrm{O}+60 \mathrm{~g} / \mathrm{l} \mathrm{H} \mathrm{SO}_{4}+0.124 \mathrm{~g} / \mathrm{l} \mathrm{NaCl}+1 \mathrm{~g} / \mathrm{l} \mathrm{PEG} 6000$ (polyethylene-glycol) + $0.0015 \mathrm{~g} / \mathrm{I}$ MPSA (3-Mercapto-1-propanesulfonic acid); (electrolyte II). 
For the preparation of the electrolytes, doubly distilled water and chemicals of p.a. quality (Sigma Aldrich Company) were used. Electrodeposition was performed using a brass of $(2.0 \times 1.0) \mathrm{cm}^{2}$ surface area as a cathode (260 1/2 hard, ASTM B36, K\&S Engineering, $250 \mu \mathrm{m}$ thick; composition: $66 \% \mathrm{Cu}, 34 \% \mathrm{Zn}$ ), while an anode was a copper foil of cylindrical shape positioned close to the wall of the cell. The electrolytes were stirred by an application of ultrasound using an ultrasonic cleaner (model HD 2200 Bandelin / Berlin, Germany) of $20 \mathrm{kHz} \pm 500 \mathrm{~Hz}$ frequency $\left(f_{\mathrm{s}}\right.$ ) equipped with a standard sonotrode (cylindrical probe of $13 \mathrm{~mm}$ tip diameter), with power (I) of $200 \mathrm{~W}$ in the continuous mode. Ultrasonic treatments were performed using amplitude $\left(P_{\mathrm{i}}\right)$ of 10,30 and $50 \%$ maximal value. Since the power of the ultrasonic device was $200 \mathrm{~W}$, the values of $P_{\mathrm{i}}$ of 10,30 and $50 \%$ corresponded to a power of 20,60 and $100 \mathrm{~W}$, respectively. The volume of the electrolyte was $100 \mathrm{ml}$ with an immersion depth of the horn of $3 \mathrm{~cm}$. For sonication of the electrolyte in the range of volumes between 20 and $900 \mathrm{ml}$, the maximum amplitude is $165 \mu \mathrm{m}$, representing $100 \%$. Working with such intensive electrolyte stirring requires temperature regulation and a system with a more adequate cooling medium. In order to mitigate temperature oscillations, electrodeposition was performed at the low temperature using ice for cooling of the electrolyte and regulation of the working temperature.

The experimental setup for Cu electrodeposition in the presence of ultrasound is shown in Figure 1.

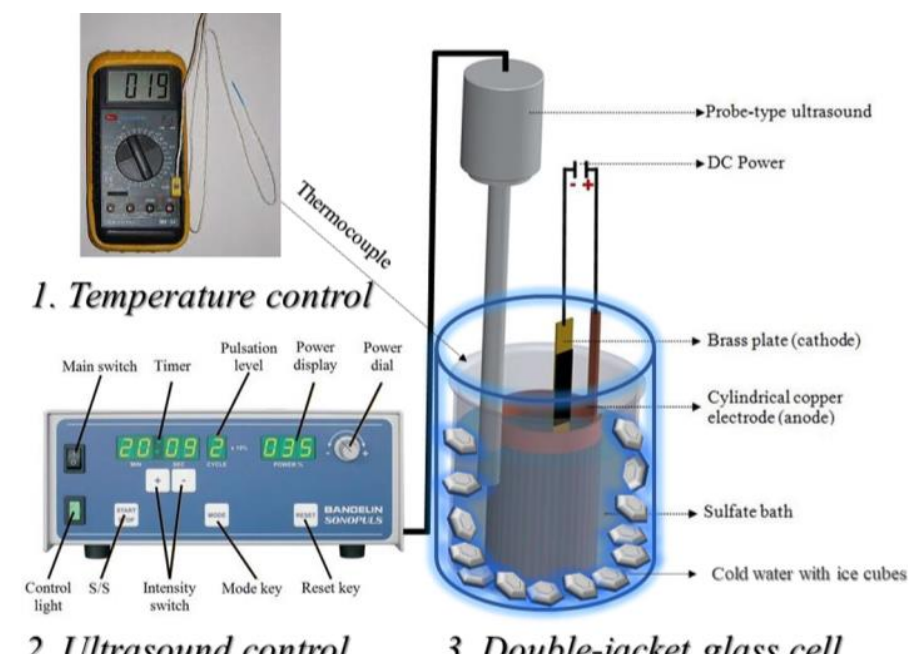

2. Ultrasound control 3. Double-jacket glass cell

Figure 1. The experimental setup for electrodeposition in the presence of ultrasound

The obtained Cu coatings were characterized by scanning electron microscopy (SEM) and atomic force microscopy (AFM) techniques.

For morphological analysis, SEM microscope model JEOL JSM-6610LV (JEOL Ltd., Tokyo, Japan) equipped with energy-dispersive X-ray spectroscopy (EDS) Oxford Instruments INCA was used.

The topographic analysis of coatings was investigated using an atomic force microscope in the non-contact mode (AFM-Auto Probe CP Research; TM Microscopes-Veeco Instruments, Santa Barbara, CA, USA). The scan area was $(20 \times 20) \mu \mathrm{m}^{2}$. Histogram analysis of the coatings was done by an application of accompanying AFM software.

Hardness analysis of the coatings was done by Vickers microindentation with applied loads between 0.049 and $2.452 \mathrm{~N}$, and a dwell time of $25 \mathrm{~s}$. Vickers microhardness tester Leitz Kleinert Prufer DURIMET I (Leitz, Oberkochen, Germany) was used for the determination of hardness.

The measured (or composite) hardness of the coating is defined by Eq. (1) [9]:

$$
H_{\mathrm{c}}=1.8544 \cdot \frac{P}{d^{2}}
$$


In Eq. (1), $H_{\mathrm{c}}$ (in $\mathrm{Pa}$ ) is a measured or composite hardness, $P$ (in $\mathrm{N}$ ) is an applied load, and $d$ (in $\mathrm{m}$ ) is a size of diagonal made in a coating during indentation. The hardness of the cathode (brass) was determined by the PSR (Proportional Specimen Resistance) model [24], and a value of 1.41 GPa [25] was obtained. The $H_{c}$ values usually include contributions of hardness both cathode (substrate) and coating, causing a necessity of application of composite hardness models for a determination of absolute (or real) hardness of coating $[9,10,25]$.

\section{Results and discussion}

Morphological and hardness analyses of the Cu coatings obtained from the basic sulphate electrolyte (electrolyte I)

Figure 2 shows morphologies and EDS spectrums of $\mathrm{Cu}$ coatings obtained at a current density of $50 \mathrm{~mA} \mathrm{~cm}^{-2}$ from the basic sulphate electrolyte with applied intensities of ultrasound corresponding to $P_{\mathrm{i}}$ of $10 \%$ (i.e. $10 \%$ of maximum ultrasound amplitude) (Figure $2 \mathrm{a}$ and $\mathrm{b}$ ) and $50 \%$ (Figure $2 \mathrm{c}$ and d). The coatings are fine-grained and similar to each other at the macro level, with visible boundaries among grains. According to expectations, EDS analyses of the obtained coatings identified only the presence of copper.

a)

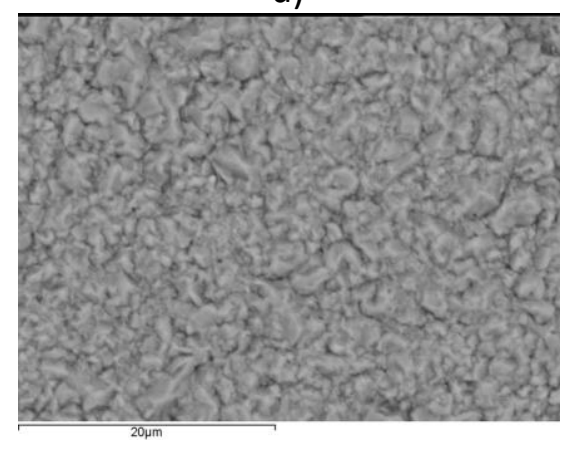

c)

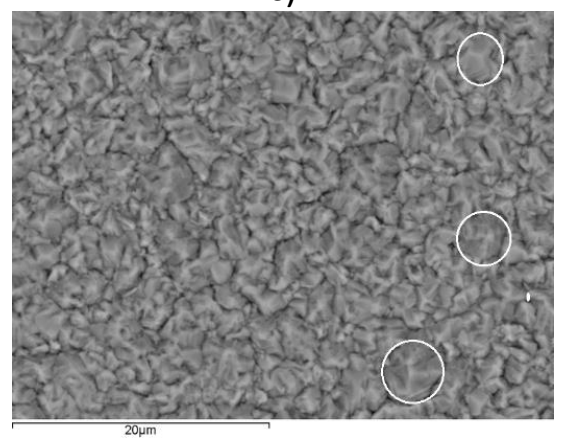

b)

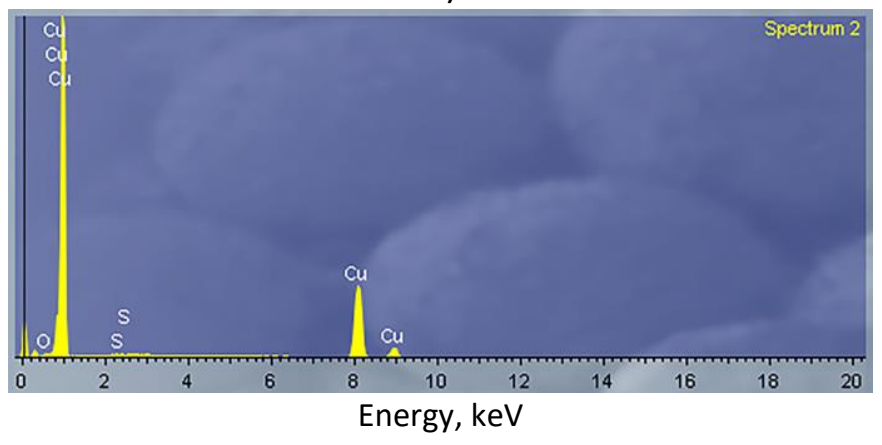

d)

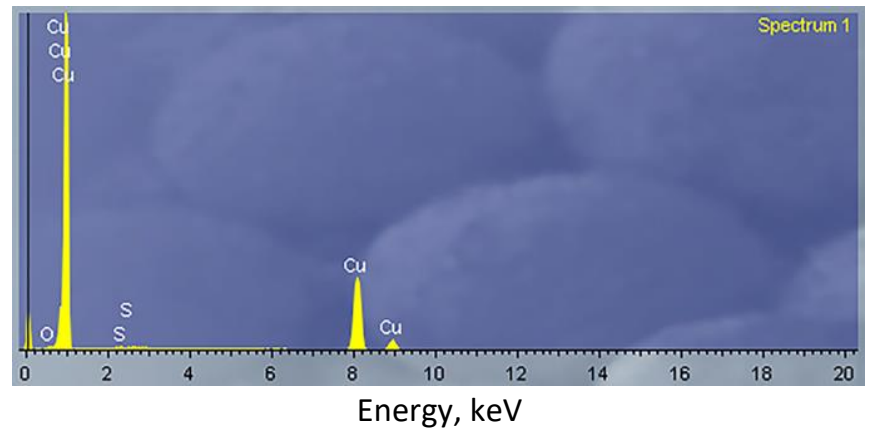

Figure 2. Morphology and EDS spectrum of copper coatings obtained at a current density of $50 \mathrm{~mA} \mathrm{~cm}^{-2}$ from electrolyte I with an intensity of ultrasound corresponding to: (a) and (b) $\mathrm{P}_{i}=10 \%$, (c) and (d) $\mathrm{P}_{i}=50 \%$

The closer analysis of structural characteristics, but with included Cu coating obtained with an intensity of ultrasound corresponding to $P_{\mathrm{i}}$ of $30 \%$, was done applying the AFM technique. Figure 3 shows the 3D (three-dimensional) images and the corresponding histograms of topography obtained with an intensity of ultrasound of $10 \%$ (Figure $3 a$ and b), $30 \%$ (Figure $3 c$ and d) and $50 \%$ (Figure 3e and f). The values of the arithmetic average of the absolute $\left(R_{\mathrm{a}}\right)$ roughness for the given Cu coatings are given in Table 1.

The increase of roughness of the coatings with increasing the intensity of applied ultrasound is noticed by analysis of data given in Table 1. 
a)

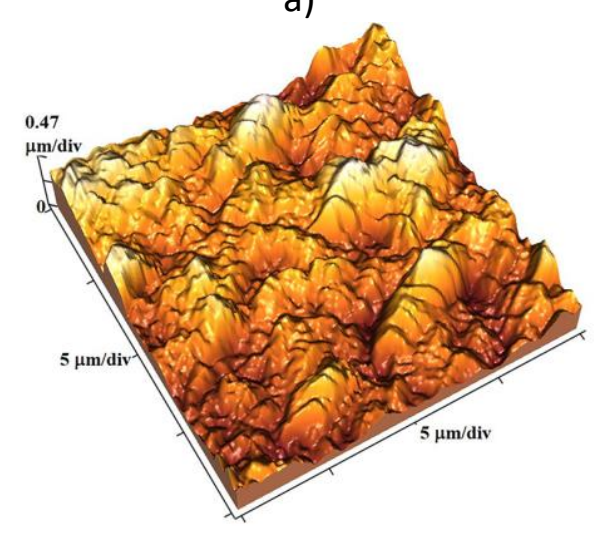

c)

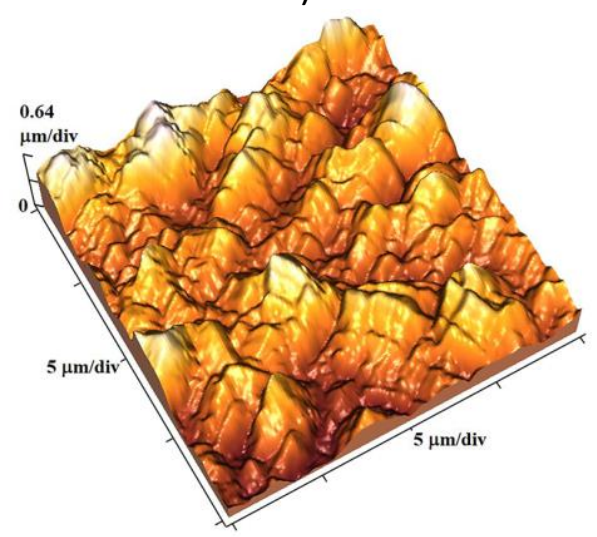

e)

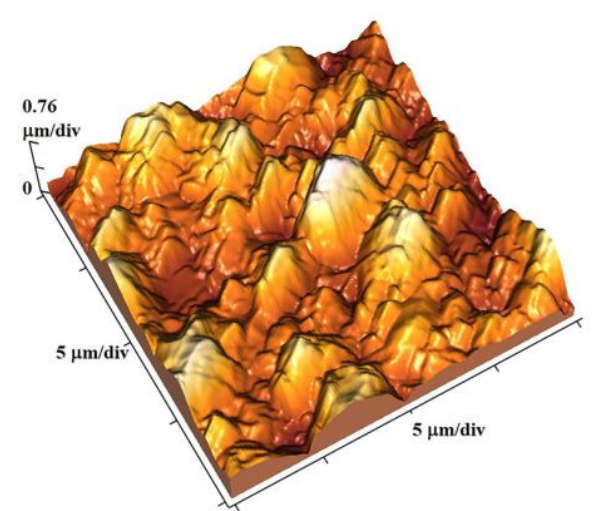

b)

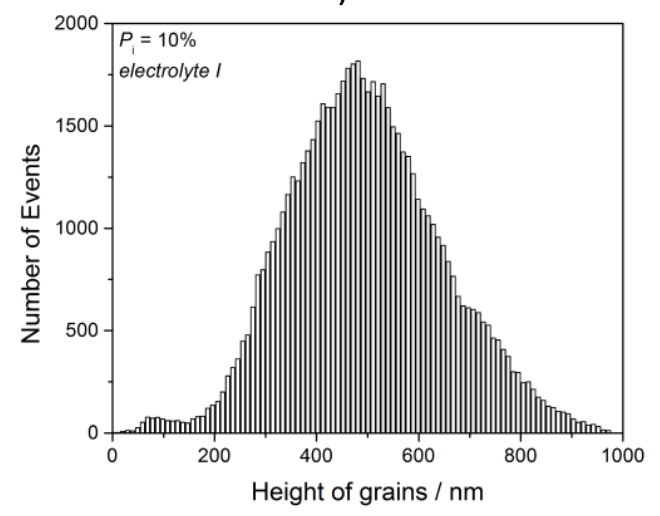

d)

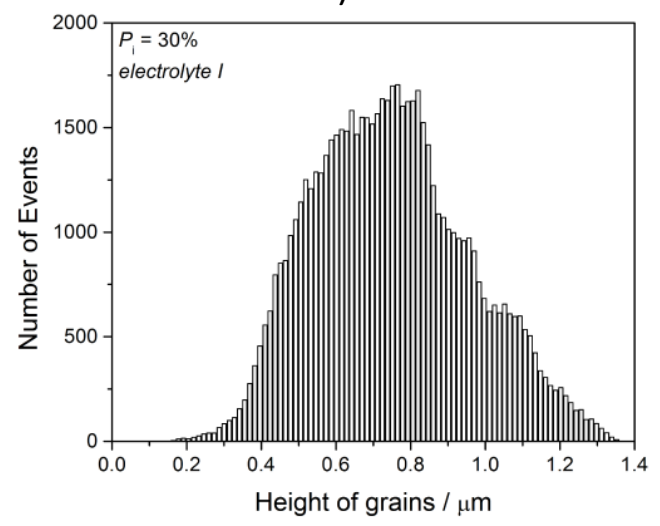

f)

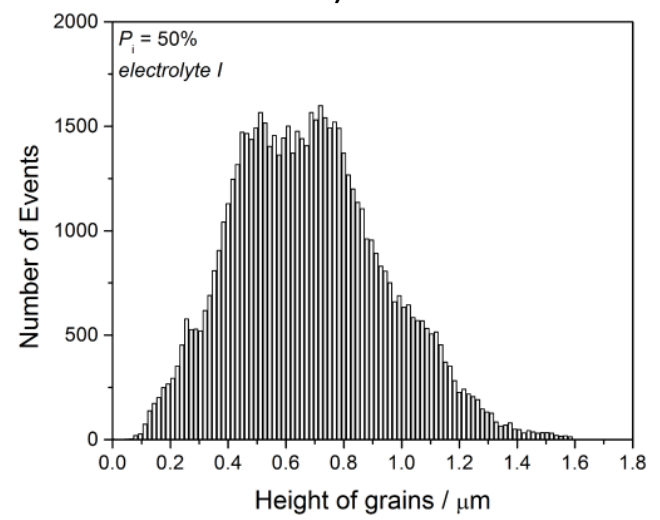

Figure 3. The 3D (three dimensional) images (left) and histograms of topography (right) for the Cu coatings obtained at $50 \mathrm{~mA} \mathrm{~cm}^{-2}$ from the basic sulphate electrolyte with an intensity of ultrasound corresponding to: (a) and (b) $\mathrm{P}_{i}=10 \%$, (c) and (d) $\mathrm{P}_{i}=30 \%$ and (e) and (f) $\mathrm{P}_{i}=50 \%$

Table 1. The values of the arithmetic average of the absolute $\left(\mathrm{R}_{a}\right)$ roughness and Meyer's index $(\mathrm{m})$ for the $\mathrm{Cu}$ coatings electrodeposited from the basic sulphate electrolyte with various intensities of applied ultrasound ( $\left.\mathrm{P}_{i}\right)$

\begin{tabular}{cccc}
\hline$P_{\mathrm{i}} / \%$ & 10 & 30 & 50 \\
\hline$R_{\mathrm{a}} / \mathrm{nm}$ & 138.1 & 181.2 & 212.4 \\
\hline$m$ & 0.4503 & 0.4594 & 0.4750 \\
\hline
\end{tabular}

The dependencies of measured composite hardness $\left(H_{c}\right)$ on the RID (relative indentation depth) for the given coatings are shown in Figure 4a. The relative indentation depth (RID) is defined by the 
ratio between the depth of an indentation $(h)$ and thickness of a coating $(\delta)$, i.e. as RID $=h / \delta$ [9]. A depth of indentation, $h$ is related with a diagonal size, $d$ as $h=d / 7$. With an application of loads above some critical value, a substrate (cathode) commences to contribute to a composite hardness, and for that reason, it is necessary to apply a composite hardness model for a determination of the absolute (or real) hardness of the coating. For electrolytically produced Cu coating/brass systems, the Chicot-Lesage (C-L) composite hardness model [26-28] showed very valuable for a determination of the absolute hardness of Cu coatings $[9,10,25,29]$. A detailed description of the C$L$ model is given in Ref. [9].

a)

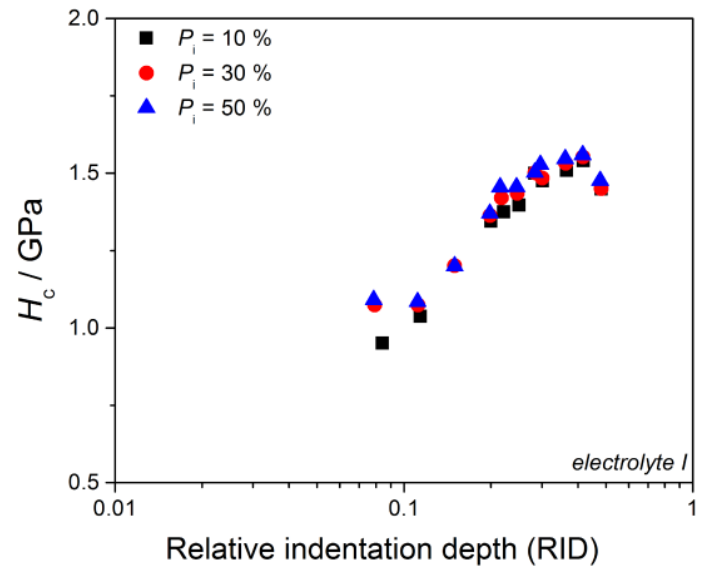

b)

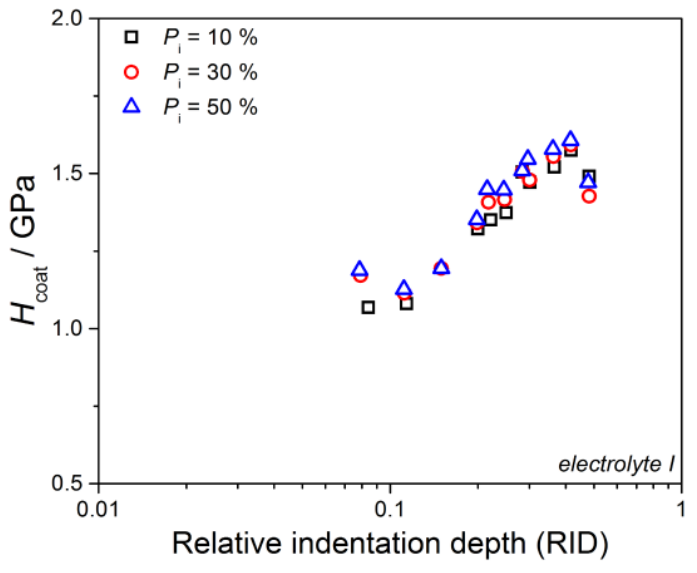

c)

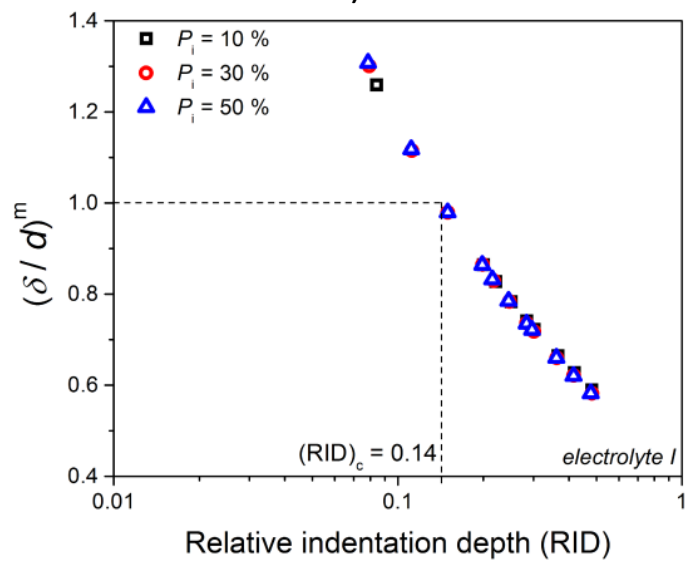

Figure 4. The dependencies of: (a) the composite $\left(\mathrm{H}_{c}\right)$ and (b) the calculated coating $\left(\mathrm{H}_{\text {coat }}\right)$ hardness on the RID for the Cu coatings electrodeposited from the basic sulphate electrolyte with various intensities of ultrasound, and (c) the dependencies of $(\delta / \mathrm{d})^{\mathrm{m}}$ on the RID for the same Cu coatings

The calculated coating hardness $\left(H_{\text {coat }}\right)$ values by the application of the C-L composite hardness model on the RID are given in Figure $4 \mathrm{~b}$. It can be clearly seen from Figure $4 a$ and $b$ that the dependencies of both $H_{c}$ and $H_{\text {coat }}$ on the RID have " $\mathrm{S}$ " shapes. Both coating hardness values grow with increasing the RID value reaching maximum at about 0.40 , and then, the sharp fall occurred approaching the values of both $H_{c}$ and $H_{\text {coat }}$ to a value of hardness of brass substrate $\left(H_{s}\right)$ of $1.41 \mathrm{GPa}$ [25].

The beginning of a contribution of the substrate to composite hardness is estimated by a determination of the dependencies of the $(\delta / d)^{\mathrm{m}}$ - the RID, where $m$ is a composite Meyer's index calculated by a linear regression performed on all experimental points for the given coating- cathode (substrate) systems $[9,24]$. The calculated $m$ values are added to Table 1 , while the dependencies of the $(\delta / d)^{m}$ - the RID are given in Figure 4c. Taking into consideration that the C-L model is valid up to 
$(\delta / d)^{m}=1$ [26-29], the critical or limiting RID value ((RID)c) of 0.14 was obtained (see Figure 4c). For RID values above 0.14 (RID $\geq 0.14$ ), a substrate (brass) significantly commences to contribute to composite (measured) hardness, and then, the calculated values obtained by application of the C-L model correspond to the absolute (or real) hardness of the $\mathrm{Cu}$ coatings. For RID values below 0.14 (RID < 0.14), an absolute hardness of the Cu coatings corresponds to the measured (composite) hardness.

Morphological and hardness analyses of the Cu coatings obtained from the electrolyte with levelling/brightening additives (electrolyte II)

The morphology and EDS spectrums of the Cu coatings obtained at a current density of $50 \mathrm{~mA}$ $\mathrm{cm}^{-2}$ from the electrolyte containing levelling and brightening additives with $P_{\mathrm{i}}$ of 10 and $50 \%$ are shown in Figure 5. At first sight, certain differences among them are visible. The coating obtained with $P_{\mathrm{i}}$ of $10 \%$ is very smooth without visible boundary among grains (Figure $5 \mathrm{a}$ ), while that obtained with an intensity of $50 \%$ can be characterized as fine-grained since certain boundaries among grains can be noticeable (Figure 5c). In both coatings, EDS spectrums identified only the presence of copper (Figure $5 b$ and $d$ ).

a)

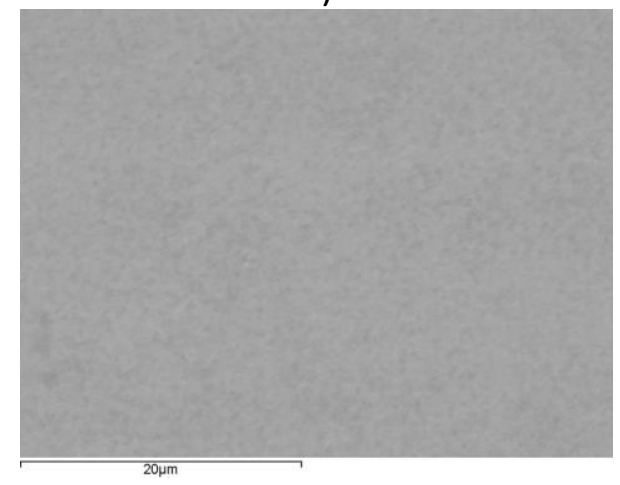

c)

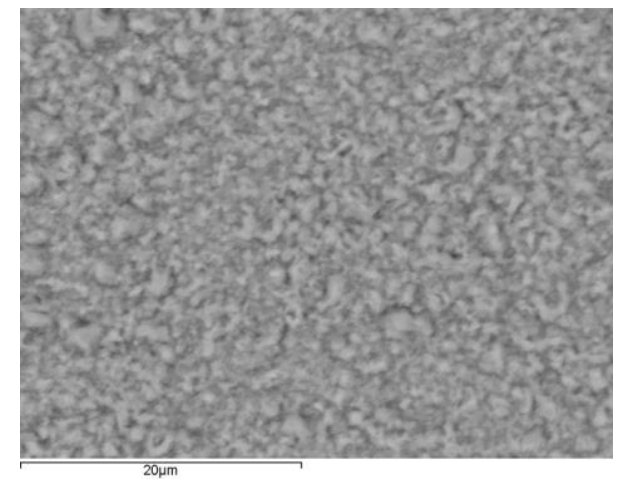

b)

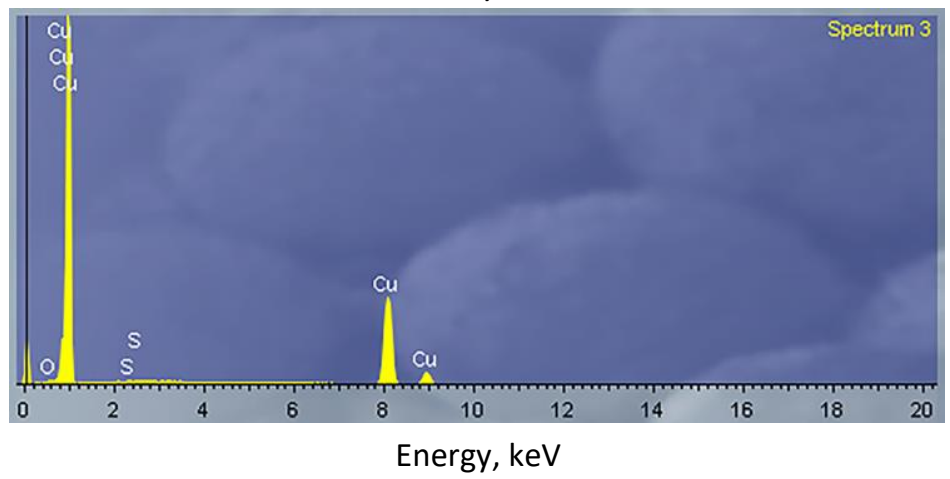

d)

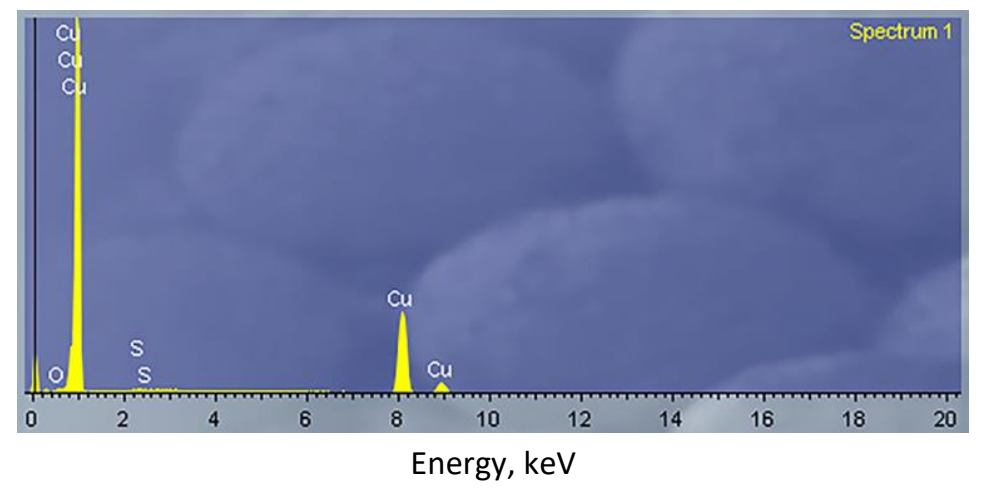

Figure 5. Morphology and EDS spectrum of copper coatings obtained at a current density of $50 \mathrm{~mA} \mathrm{~cm} \mathrm{crom}^{-2}$ from electrolyte II with an intensity of ultrasound corresponding to: (a) and (b) $\mathrm{P}_{i}=10 \%$, (c) and (d) $\mathrm{P}_{i}=50 \%$

The AFM analysis, including the 3D AFM images and histograms of the topography, for the $\mathrm{Cu}$ coatings obtained with an intensity of ultrasound of 10,30 and $50 \%$ is shown in Figure 6 . The values of $R_{a}$ roughness for these coatings are given in Table 2 .

Similar to the $\mathrm{Cu}$ coatings obtained from the basic sulphate electrolyte, the roughness of the $\mathrm{Cu}$ coatings obtained from the electrolyte with additives increased with increasing the intensity of ultrasound. 
a)

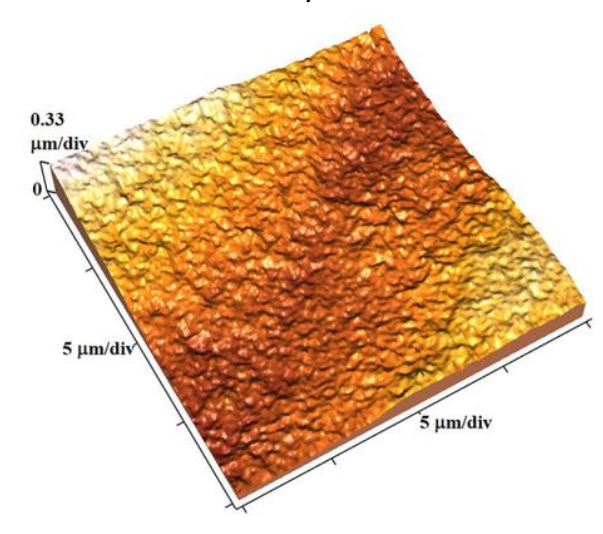

c)

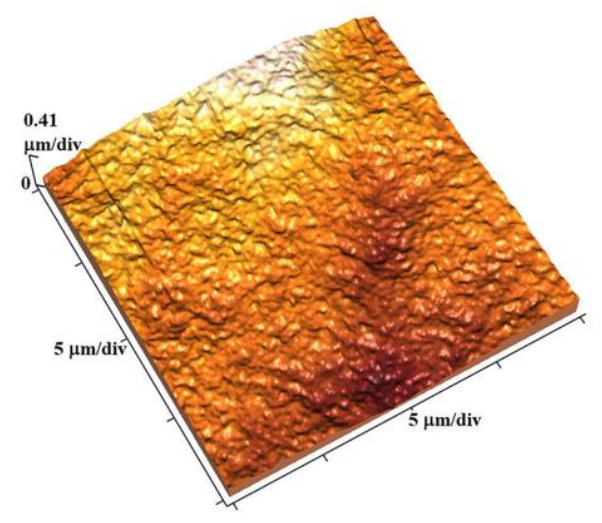

e)

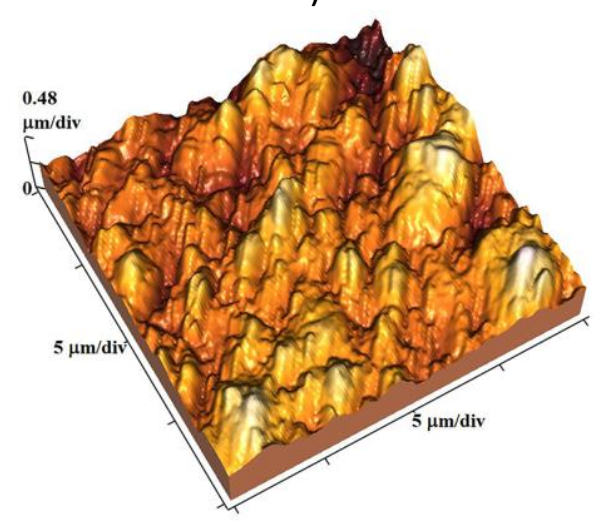

b)

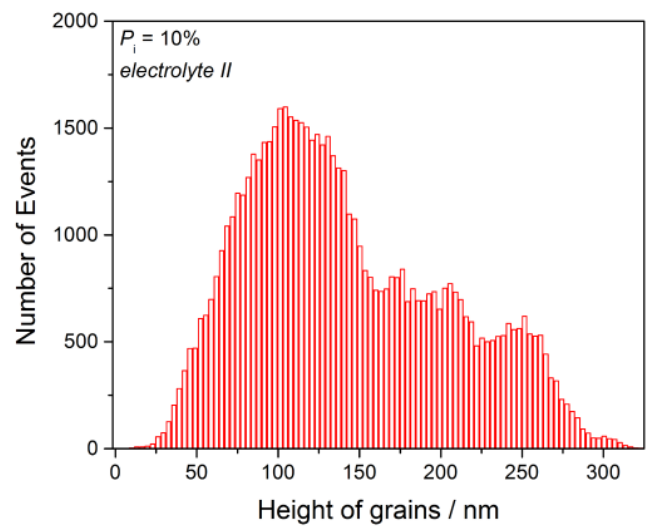

d)

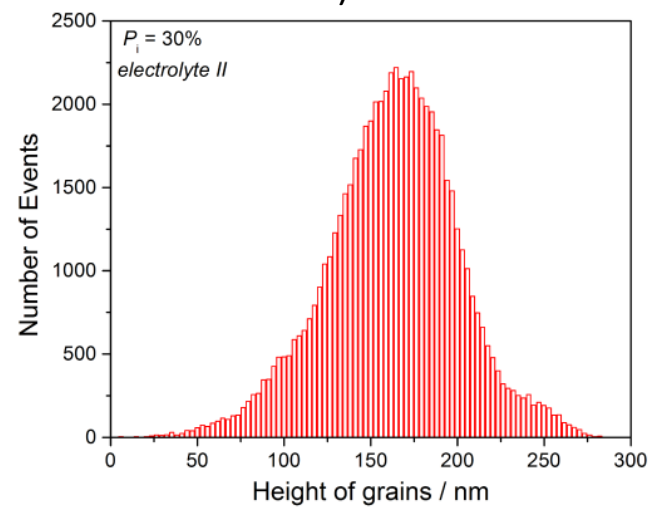

f)

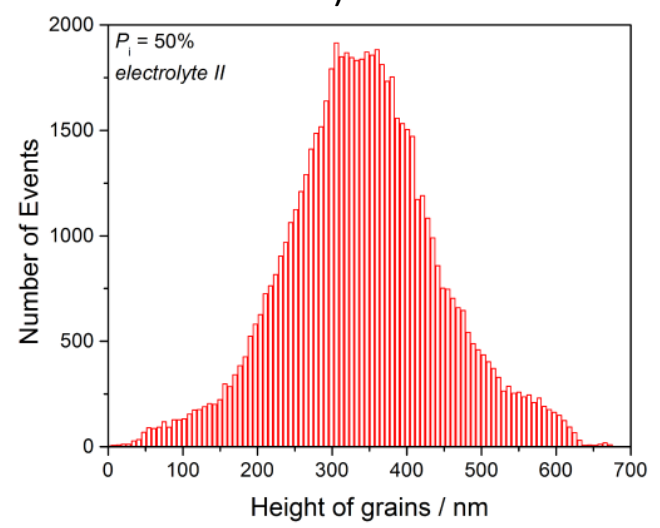

Figure 6. The $3 D$ (three dimensional) images (left) and histograms of topography (right) for Cu coatings obtained at $50 \mathrm{~mA} \mathrm{~cm}^{-2}$ from the electrolyte containing levelling/brightening additives with an intensity of ultrasound corresponding to: (a) and (b) $\mathrm{P}_{i}=10 \%,(c)$ and $(d) \mathrm{P}_{i}=30 \%$ and $(e)$ and $(f) \mathrm{P}_{i}=50 \%$

Table 2. The values of the arithmetic average of the absolute $\left(R_{a}\right)$ roughness and Meyer's index $(m)$ for the Cu coatings electrodeposited from the electrolyte with added additives for levelling/brightening in the presence of various intensities of ultrasound.

\begin{tabular}{cccc}
\hline$P_{\mathrm{i}} / \%$ & 10 & 30 & 50 \\
\hline$R_{\mathrm{a}} / \mathrm{nm}$ & 26.87 & 53.07 & 90.5 \\
\hline$m$ & 0.4407 & 0.4565 & 0.4725 \\
\hline
\end{tabular}

The dependencies of the measured (composite) hardness $\left(H_{c}\right)$ and the calculated coating hardness $\left(H_{\text {coat }}\right)$ by the C-L model on the RID for the $\mathrm{Cu}$ coatings obtained from the electrolyte- 
containing additives are shown in Figures 7a and b, respectively. The " $\mathrm{S}$ " shape of curves is not mentioned in this case. The probable reason for it lies in the fact that a value of a brass hardness of $1.41 \mathrm{GPa}$ was not exceeded for these coatings. Anyway, it is necessary to note that $H_{\mathrm{c}}$ and $H_{\text {coat }}$ values for the given coatings were smaller than those obtained from the additive-free electrolyte.

a)

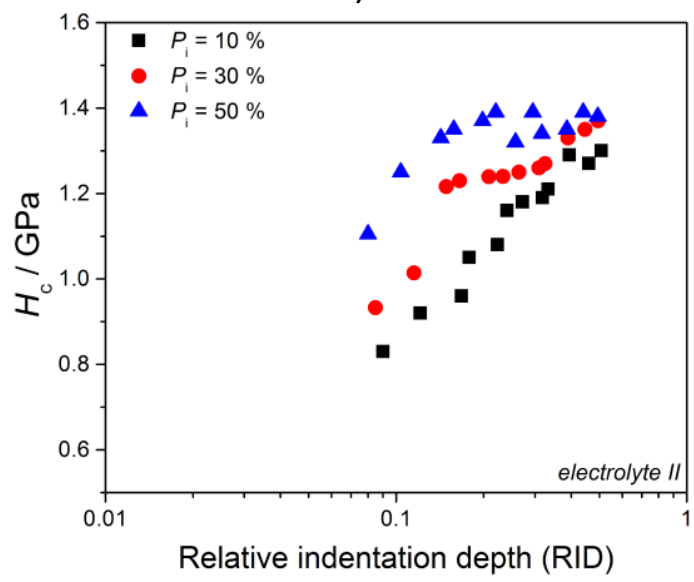

b)

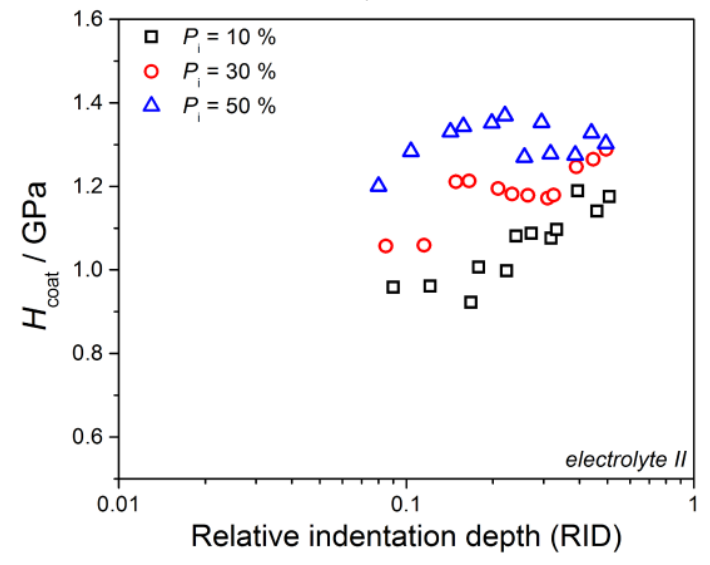

c)

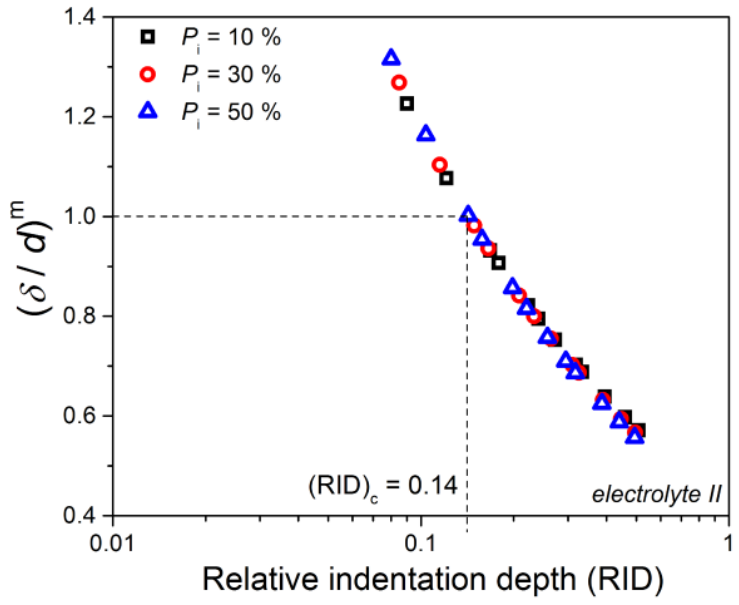

Figure 7. The dependencies of: (a) the composite $\left(\mathrm{H}_{c}\right)$ and $(b)$ the calculated coating $\left(\mathrm{H}_{\text {coat }}\right)$ hardness on the RID for the $\mathrm{Cu}$ coatings electrodeposited from the electrolyte containing the levelling/brightening additives with various intensities of ultrasound, and $(c)$ the dependencies of $(\delta / d)^{m}$ on the RID for the same Cu coatings

The dependencies of the $(\delta / d)^{\mathrm{m}}$ - the RID for the given coatings are shown in Figure $7 \mathrm{c}$, while the calculated values of $m$ index used in a determination of these dependencies are added to Table 2 . Using the same procedure as applied for the Cu coatings obtained from the additive-free electrolyte, the limiting or critical RID value ((RID)c) of 0.14 , separating the zone in which the composite hardness can be equalled with the coating hardness and the zone requiring an application of the $C-L$ model for a determination of the absolute hardness of the Cu coatings, was also obtained.

\section{Discussion of the presented results}

Formation of morphologies of $\mathrm{Cu}$ coatings obtained from electrolyte I and II with various intensities of ultrasound can be explained as follows: a current density of $50 \mathrm{~mA} \mathrm{~cm}^{-2}$ belongs to the mixed activation-diffusion control of the electrodeposition [9]. In this control of the electrodeposition, deposition overpotential, $\eta$ is given by Eq. (2) $[4,30]$ :

$$
\eta=\frac{b_{c}}{2.3} \ln \frac{j}{j_{0}}+\frac{b_{c}}{2.3} \ln \frac{1}{1-\left(j / j_{\mathrm{L}}\right)}
$$


where $b_{c}$ is the cathodic Tafel slope, $j_{0}$ is the exchange current density and $j_{L}$ is the limiting diffusion current density.

The deposition overpotential consists of the activation ( $\left.\eta_{\text {act }}\right)$ and the diffusion ( $\left.\eta_{\text {diff }}\right)$ parts. The activation part ( $\eta_{\text {act }}$ ) required for the charge transfer is presented by Eq. (3) $[4,30]$ :

$$
\eta_{\text {act }}=\frac{b_{\mathrm{c}}}{2.3} \ln \frac{j}{j_{0}}
$$

while the diffusion part ( $\left.\eta_{\text {diff }}\right)$ is given by Eq. (4) [4,30]:

$$
\eta_{\text {diff }}=\frac{b_{c}}{2.3} \ln \frac{1}{1-\left(j / j_{L}\right)}
$$

and it is due to mass transfer limitations (diffusion limitation primarily).

The imposed ultrasound only affects the diffusion part of deposition overpotential. The imposed ultrasound causes a stirring of electrolyte in the near-electrode layer leading to a decrease of diffusion layer thickness and up to an increase of the limiting diffusion current density. This process leads to a decrease of diffusion part in overall deposition overpotential, i.e. up to a decrease of the degree of diffusion control in the mixed activation-diffusion control. This decrease enhances with an increase of intensity of applied ultrasound.

The concept of "effective overpotential" can be proposed to explain the change of surface morphology under an imposed ultrasound. This concept was originally proposed to explain the morphology of Cu deposits formed under vigorous hydrogen evolution [31]. In conditions of vigorous hydrogen evolution, the electrodeposition process occurs at overpotential, which is effectively lower than the specified value, and this overpotential is denoted as "effective" value. According to this concept, vigorous hydrogen evolution causes a stirring of the electrolyte in the near-electrode layer, causing the decrease of thickness of diffusion layer, the increase of the limiting diffusion current density, and as a result of this process, the decrease of the degree of diffusion control of electrodeposition. From the morphological point of view, it means that morphologies of metal deposits become similar to those obtained at the lower overpotentials at which there is no hydrogen evolution, or it is very small.

This concept applies to all cases where there is a change of hydrodynamic conditions in the nearelectrode layer, like the case of imposed magnetic fields [30]. Then, the effect of various intensities of ultrasound on the morphology of Cu coatings (Figures 2,3,5 and 6) can be considered as follows: with an increase of intensity of applied ultrasound, it intensifies an electrolyte stirring in the nearelectrode layer, enhancing the decrease of the diffusion layer thickness and the increase of the limiting diffusion current density. Hence, the larger intensity of ultrasound, the smaller degree of diffusion control is. In Eq. (2), as already mentioned, the activation part does not depend on whether ultrasound is imposed to the electrodeposition process or not. With the decreasing diffusion part in Eq. (2) caused by the application of ultrasound, it increases the contribution of activation control in the mixed activation-diffusion control. This can be easily confirmed by analysis morphologies of $\mathrm{Cu}$ coatings obtained from the basic sulphate electrolyte (Figure $2 \mathrm{a}$ and $\mathrm{c}$ ). The larger contribution of the activation control in the mixed activation-diffusion control in the coating obtained with the larger intensity of imposed ultrasound (Figure 2c) can be proved by appearing of the larger and welldefined grains in this coating (see parts in circles in this figure). Namely, it is well known [4] that large and regular grains are characteristic of the activation control of electrodeposition.

A similar effect on the morphology of copper coatings is observed by the regime of pulsating current (PC) regime [9]. Analysing the copper coatings obtained at various average current densities, 
which belonged to the mixed activation-diffusion control, formation of coatings with larger and well defined crystal grains was observed at the average current density which corresponded to the very beginning of the activation-diffusion control. The formation of coatings with such characteristics is explained by the increase of ratio of activation control in the mixed activation-diffusion control of electrodeposition with decreasing the average current density.

Although certain differences in morphology of Cu coatings obtained under various intensities of ultrasound are obtained, a noticeable difference in their hardness is only observed between the coatings obtained from the basic sulphate electrolyte and those obtained from the electrolyte with additives. The absence of any noticeable difference with various intensities of ultrasound can be attributed to small differences in the surface morphology reached by application of ultrasound, as well as to a sensitivity of the Vickers microindentation technique used for a determination of the coating hardness. Namely, for the applied loads in the $(0.049-2.452 \mathrm{~N})$ range, the size of the diagonal of indent was in the $(9.78-56.02 \mu \mathrm{m})$ range. Analysing morphologies of Cu coatings shown in Figures 2, 3, 5 and 6, it is clear that these surface morphologies were not significantly sensitive for this method of a determination of coating hardness.

Anyway, the critical or limiting RID value ((RID) $)$ of 0.14 , separating the zone in which the composite hardness can be equalled with the coating hardness (negligible effect of the cathode hardness on the composite hardness) and the zone of a necessary application of the $C-L$ model for a determination of the absolute hardness of the Cu coatings (the strong effect of the cathode hardness on the composite hardness) was confirmed for all analysed $\mathrm{Cu}$ coatings. The fact that this value was obtained for the Cu coatings produced by various regimes of electrodeposition, such as the pulsating current (PC) [9,32] and the constant galvanostatic (DC) [10] regimes, as well as by varying all relevant parameters of electrodeposition in the DC regime, such as the type of cathode [10], the thickness of coating [25], the type of electrolyte [10] and kind of electrolyte stirring [10] clearly indicates that (RID)c value of 0.14 has universal character for electrolytically produced copper coatings.

\section{Conclusions}

The $\mathrm{Cu}$ coatings produced by the DC regime from electrolytes without and with additives for levelling and brightening were characterized by SEM and AFM techniques (surface characterization) and by Vickers microindentation (mechanical characterization). The various intensities of ultrasound were used for the electrolyte stirring. On the basis of the obtained results, it follows:

- The increasing intensity of ultrasound worsens the quality of the coatings. It is manifested by the increase of roughness of coatings, and it is valid for both types of Cu coatings, i.e. for those obtained from the electrolytes both without and with added additives.

O The change in the quality of coatings is explained by a strong effect of ultrasonic waves on hydrodynamic conditions in the near-electrode layer. Increasing the intensity of ultrasound, it increases a share of the activation control in the mixed activation-diffusion control, which is accompanied by the formation of larger and well-defined grains.

- Mathematical set equations are used to explain a change of surface morphology under the influence of ultrasonic waves. It is shown that the concept "effective overpotential" originally proposed for copper electrodeposition under vigorous hydrogen evolution can be also applied for Cu electrodeposition under imposed ultrasonic stirring of the electrolyte.

- The hardness of $\mathrm{Cu}$ coatings obtained from the electrolyte without additives was larger than those obtained from the electrolyte with additives. The use of various intensities of ultrasound did not significantly affect hardness of the coatings, especially for those obtained from the basic sulphate electrolyte. 
Acknowledgment: This work was funded by Ministry of Education, Science and Technological Development of Republic of Serbia (Grants No. 451-03-68/2022-14/200026 and 451-03-68/202214/200135).

\section{References}

[1] Copper plating service, https://www.sharrettsplating.com/coatings/copper (accessed on 27 September 2021).

[2] N. Bharadishettar, U. K. Bhat, D. B. Panemangalore, Metals 11 (2021) 711. https://doi.org/ 10.3390/met11050711

[3] Z. Tan, S. Liu, J. Wu, Z. Nan, F. Yang, D. Zhan, J. Yan, B. Mao, ChemElectroChem (2022) e202101412. https://doi.org/10.1002/celc.202101412.

[4] K. I. Popov, S. S. Djokić, N. D. Nikolić, V. D. Jović. Morphology of Electrochemically and Chemically Deposited Metals, Springer International Publishing, New York, USA, 2016, https://doi.org/10.1007/978-3-319-26073-0.

[5] N. D. Nikolić, G. Branković, M. G. Pavlović, Journal of Electrochemical Science and Engineering 2 (2012) 33-40. https://doi.org/10.5599/jese.2012.0009.

[6] N. D. Nikolić, Journal of Electrochemical Science and Engineering 10 (2020) 111-126. http://dx.doi.org/10.5599/jese.707.

[7] Y. H. Zhang, M. Z. An, P. X. Yang, J. Q. Zhang, Electrocatalysis 12 (2021) 619-627. https://doi.org/10.1007/s12678-021-00687-2.

[8] R. Carpio, A. Jaworski, Journal of The Electrochemical Society 166 (2018) 3072-3096. https://doi.org/10.1149/2.0101901jes.

[9] I. O. Mladenović, J. S. Lamovec, D. G. Vasiljević Radović, R. Vasilić, V. J. Radojević, N. D. Nikolić, Metals 10 (2020) 488. https://doi.org/10.3390/met10040488.

[10] I. O. Mladenović, J. S. Lamovec, D. G. Vasiljević-Radović, R. Vasilić, V. J. Radojević, N. D. Nikolić, Metals 11 (2021) 1807. https://doi.org/10.3390/met11111807.

[11] A. Tamilvanan, K. Balamurugan, K. Ponappa, B. Madhan Kumar, International Journal of Nanoscience 13 (2014) 1430001. https://doi.org/10.1142/S0219581X14300016.

[12] L. Martins, J. Martins, A. Romeira, M. E. V. Costa, J. S. Costa, M. Bazzaoui, Materials Science Forum 455-456 (2004) 844-848 https://doi:10.4028/www.scientific.net/msf.455-456.844.

[13] J. An, G. Xie, W. Xia, H. Wang, B. Ren, K. Liu, Powder Technology 386 (2021) 193-198. https://doi.org/10.1016/j.powtec.2021.03.034.

[14] S. Derbal, M. Benaicha, Journal of Electronic Materials 50 (2021) 5134-5140. https://doi.org/10.1007/s11664-021-09057-6.

[15] N. E. Tarolla, S. Voci, J. Reyes-Morales, A. D. Pendergast, J. E. Dick, Journal of Materials Chemistry A 9 (2021) 20048-20057. https://doi.org/10.1039/D1TA02369A.

[16] F. A. Lowenheim, Modern Electroplating, 3rd Edition, Wiley-Interscience, New York, USA, 1974. ISBN 13: 978-0471549680

[17] N. N. C. Isa, Y. Mohd, M. H. M. Zaki, S. A. S. Mohamad, International Journal of Electrochemical Science 12 (2017) 6010-6021 https://doi.org/10.20964/2017.07.58.

[18] X. Liang, X. Ren, R. He, T. Ma, A. Liu, New Journal of Chemistry 45 (2021) 19655-19659. https://doi.org/10.1039/D1NJ03503G.

[19] N. D. Nikolić, Z. Rakočević, K. I. Popov, Journal of Electroanalytical Chemistry 514 (2001) 5666. https://doi.org/10.1016/S0022-0728(01)00626-X.

[20] L. Bonou, M. Eyraud, R. Denoyel, Y. Massiani, Electrochimica Acta 47 (2002) 4139-4148. https://doi.org/10.1016/S0013-4686(02)00356-0

[21] Q. Chen, Z. Wang, J. Cai, L. Lui, Microelectronic Engineering 87 (2010) 527-531. https://doi.org/10.1016/i.mee.2009.06.035 
[22] Y.-Q. Wang, X.-L. Fu, W.-L. Xu, M. Li, X.-X. Zhang, The Chinese Journal of Process Engineering 4 (2004) 305-309.

[23] J. M. Costa, A. F. A. Neto, Ultrasonics Sonochemistry 68 (2020) 105193. https://doi.org/ 10.1016/i.ultsonch.2020.105193

[24] H. Li, R.C. Bradt, Materials Science and Engineering: A 142 (1991) 51-61. https://doi.org/ 10.1016/0921-5093(91)90753-A

[25] I. O. Mladenović, N. D. Nikolić, J. S. Lamovec, D. G. Vasiljević Radović, V. J. Radojević, Metals 11 (2021) 111. https://doi.org/10.3390/met11010111

[26] J. Lesage, A. Pertuz, E. S. Puchi-Cabrera, D. Chicot, Thin Solid Films 497 (2006) 232-238. https://doi.org/10.1016/i.tsf.2005.09.194

[27] J. Lesage, D. Chicot, Surface and Coatings Technology 200 (2005) 886-889. https://doi.org/ 10.1016/i.surfcoat.2005.01.056

[28] D. Chicot, J. Lesage, Thin Solid Films 254 (1995) 123-130. https://doi.org/10.1016/00406090(94)06239-H

[29] I. O. Mladenović, J. S. Lamovec, D. G. Vasiljević-Radović, V. J. Radojević, N. D. Nikolić, International Journal of Electrochemical Science 15 (2020) 12173-12191. http://dx.doi.org/ $10.20964 / 2020.12 .01$

[30] N. D. Nikolić, Journal of the Serbian Chemical Society 72 (2007) 787-797 https://doi.org/ 10.2298/JSC0709787N

[31] N. D. Nikolić, K. I. Popov, Lj. J. Pavlović, M. G. Pavlović, Journal of Electroanalytical Chemistry 588 (2006) 88-98. https://doi.org/10.1016/j.jelechem.2005.12.006

[32] I. O. Mladenović, J. S. Lamovec, D. G. Vasiljević-Radović, V. J. Radojević, N. D. Nikolić., Journal of the Serbian Chemical Society 87 (2022) 899-910 https://doi.org/10.2298/JSC2110 $\underline{14105 \mathrm{M}}$

(C2022 by the authors; licensee IAPC, Zagreb, Croatia. This article is an open-access article distributed under the terms and conditions of the Creative Commons Attribution license (https://creativecommons.org/licenses/by/4.0/) 
